\title{
The Gaseous Electronic Conference (GEC) reference cell as a benchmark for understanding microelectronics processing plasmas*
}

\author{
M. L. Brake, ${ }^{\dagger}$ J. Pender, ${ }^{\text {a) }}$ and J. Fournier ${ }^{\text {b) }}$ \\ Department of Nuclear Engineering and Radiological Sciences, University of Michigan, \\ Ann Arbor, Michigan 48109-2104
}

(Received 17 November 1998; accepted 29 January 1999)

\begin{abstract}
A collaborative experimental effort was initiated at a workshop at the 1988 Gaseous Electronics Conference (GEC) to start a program to understand the fundamental physics of processing plasmas, as well as give researchers a baseline experiment to develop plasma diagnostics to be used on manufacturing plasma systems. The design was based on the use of 4 in. diameter, aluminum electrodes in a parallel plate configuration at $13.56 \mathrm{MHz}$, run in a capacitively coupled discharge mode. Before conclusions about commercial plasma systems can be made from experimental results from the GEC cell, the GEC cell must be shown to behave similarly to that of a commercial system. The etching performance of a GEC cell was compared to a SEMI Group $1000 \mathrm{TP} / \mathrm{CC}$ reactive ion etcher (RIE). The GEC cell and the RIE gave similar etch rates and fluorine concentrations when the electrode plate spacing and power density were the same. (C) 1999 American Institute of Physics. [S1070-664X(99)95905-0]
\end{abstract}

\section{INTRODUCTION}

Plasma assisted materials processing is playing an ever increasing role in manufacturing, especially in the manufacturing of microelectronics. Plasma processing has been used since the 1970s for etching semiconductor materials using $\mathrm{CF}_{4} / \mathrm{O}_{2}$ plasmas. ${ }^{1}$ The initial advantages of plasma processing over "wet" processing with liquid chemicals were the use of safe nontoxic gases (e.g., $\mathrm{CF}_{4}$ ) rather than corrosive liquids, which were not easily disposed of, and due to simple automation of plasma processing over wet chemistry. ${ }^{1}$ Also, with "high" plasma temperatures (a few eV), electrons have enough energy to collide with the molecules so that reactive free radicals can be formed. If there were no discharge, the gas temperature would need to be very large to produce the reactive radicals needed for etching. Silicon wafers cannot withstand such high temperatures. ${ }^{1}$ Glow discharges offer the advantage of "hot" chemistries at low gas temperatures.

In subsequent years, it has turned out that there are other advantages to plasma or "dry" etching. Etching involves pattern transfer by the removal of the exposed area at the wafer surface. Plasmas can be used as a source of reactants at the wafer surface. Collisional processes in the discharge dissociate or "crack" the feed gas, producing free radicals, which then are able to react with the wafer surface. One of the most widely used etchants for silicon based devices is fluorine, due to its high reactivity with silicon. The advantage of plasma based etching is that not only is atomic fluorine available for surface reactions, but ions produced in the discharge accelerate across the plasma sheath (to be described later) that surrounds the wafer and sputter matter off

*Paper F3I2.4 Bull. Am. Phys. Soc. 43, 1705 (1998).

†'Invited speaker.

a) Applied Materials, 3050 Bowers Ave, Santa Clara, California 95054.

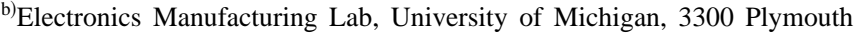
Rd, Ann Arbor, Michigan 48105. of the wafer. However, in an ideal etching situation, a combination of etchant atoms, such as reactive $\mathrm{F}$ atoms and energetic ions, produce much higher etch rates compared to chemical or sputter etching alone. ${ }^{2}$ The energetic ions transfer energy to the surface of the wafer and the chemical reaction with fluorine is enhanced. This allows for anisotropic etching. That is, "ion-enhanced energy-driven etching",2 provides vertical etching at a much faster rate than horizontal etching because of the directionality of the ions as they are accelerated across the sheath in a perpendicular direction to the wafer. Anisotropic etches allow the wall shape to be controlled and make it possible for device features to be placed more closely together. This means that more circuit elements can be patterned into a smaller space. The industry has been striving for a larger and larger number of components per circuit. Gordon Moore has observed that since 1960, the number of components per circuit has doubled every year. ${ }^{1}$

Plasma etching offers many benefits to the microelectronics manufacturing industry, but it has also introduced complicated issues, such as defects caused by ion bombardment. ${ }^{1}$ As plasma processing has become predominant in industry, it has become necessary to understand the underlying plasma physics and plasma chemistry to improve and control the processes. As the semiconductor industry moves toward automating each fabrication step, a need for a more complete understanding of not only the discharges used but also the behavior of the tools, i.e., the plasma etchers, is needed, especially if the process in going to be remotely controlled.

One of the most commonly used modes of operations is a capacitively driven radio frequency (rf) discharge, sometimes referred to as a radio frequency (rf) diode. ${ }^{2}$ Most of the rf discharge processes operate at $13.56 \mathrm{MHz}$. This is the frequency given to the plasma processing industry by the international communications authorities. ${ }^{3}$ In the capaci- 


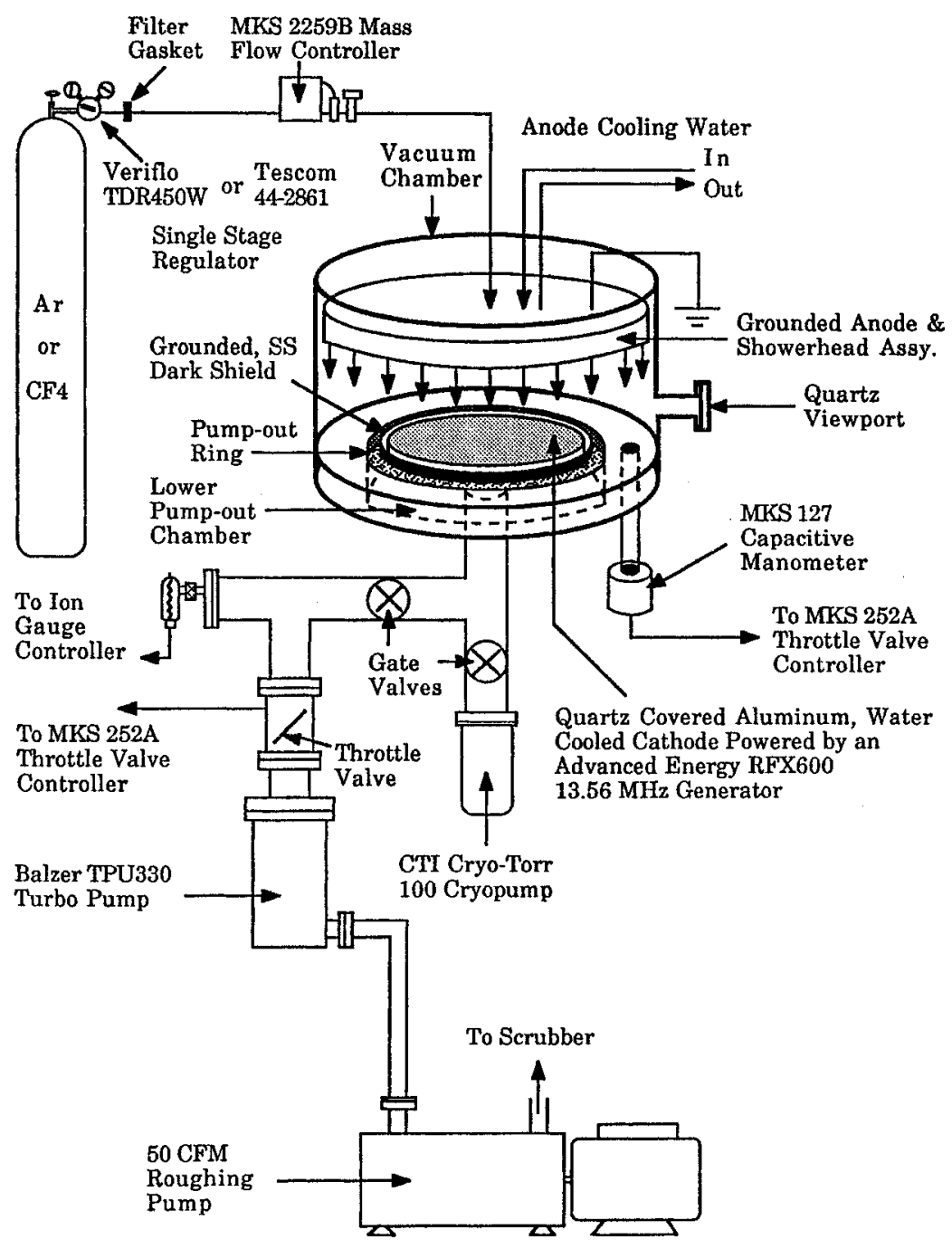

FIG. 1. Schematic of the SEMI Group RIE.

\section{SEMI Group Parallel Plate RIE}

tively coupled mode, the upper electrode is grounded and the wafer is placed on the lower, powered, electrode. Sometimes this configuration is known as a reactive ion etcher (RIE). A capacitively coupled discharge that operates at low pressures (below 1 Torr) is also referred to as RIE. However, reactive ion etching, "by definition should be used for etching by ions that react with and remove substrate material." 1 See Fig. 1 for a schematic of the SEMI Group 1000 TP/CC capacitively coupled, reactive ion etcher.

Since no direct current can flow out from the cathode, a negative bias voltage develops. Because the frequency of the applied voltage is very fast, the heavy ions cannot respond as quickly as the electrons on the same time scale as the rf power oscillations. During the first few rf cycles, the flux of electrons to the cathode is much greater than the flux of ions, which causes a net negative charge to develop. Eventually this dc bias voltage or dc offset is large enough that the cathode spends most of the rf cycle at a negative voltage. Equilibrium is reached when the flux of positive charges to the surface is balanced by the negative charge flux. This bias voltage accelerates positive ions to the surface increasing the etch rates and anisotropy. The current density at the sheath is space charge limited with flux $J$ such that the flux at the sheath at the sheath is proportional to

$$
J \propto \frac{V^{3 / 2}}{l^{2}},
$$

where $J$ is the current flux density across the sheath, $V$ is the voltage, and $l$ is the sheath thickness. ${ }^{2,3}$ Equation (1) is the Child law of space-charge-limited current in a plane diode. ${ }^{2}$ Note that this simple model of the sheath does not include collisions. However, if the momentum transfer is independent of ion velocity, then the ion flux still scales with the voltage as $V^{3 / 2}$, although the sheath thickness dependence is different. $^{2}$ This voltage scaling should be a reasonable estimate for the experiments presented here as the pressure is quite low, in the millitorr range, and the plasma is not highly collisional.

Plasmas used in manufacturing of microelectronics are very complicated and difficult to understand. The plasma chambers used in manufacturing vary in geometry and size, 
are made of different materials, and vary in operating conditions such as power and pressure. Because of these different machine variations, it is difficult to develop one model to describe all plasma tools, even if the plasma systems are all used to perform the same function, e.g., etching silicon in the manufacturing of microelectronics.

At the 1988 Gaseous Electronic Conference (GEC) meeting, a collaborative experimental effort was initiated at a workshop on the "Design, Calibration and Modeling of RF Plasma Processing Systems." It was agreed that there needed to be an easy to model "Reference Cell" for making comparative measurements with other "identical" systems. The design was based upon the use of 4 in. diameter, water cooled, aluminum electrodes in a parallel plate configuration with a showerhead gas inlet, to be run in a reactive ion etching mode, i.e., capacitively coupled mode. Researchers from Sandia National Labs developed the blueprints for the Reference Cell and, initially, five institutions built, tested, and compared basic voltage and current measurements. ${ }^{4}$ Since then many researchers have built GEC Reference Cells. A preliminary report of the etching properties of the GEC cell has been reported in Ref. 5. In recent years, some researchers have retrofitted the top electrode with an inductively coupled coil so that a higher density plasma can be obtained (see Ref. 6) and the dc bias voltage across the sheath can be independently controlled by a capacitively coupled rf source.

Another goal of building a GEC Reference Cell was to study the plasma physics of the etching chemistries, as well as to develop diagnostics to be used on commercial plasma systems. The University of Michigan has concentrated on comparing the results of the performance of the GEC Reference Cell to those of commercial tools and to develop and test sensors on the user friendly GEC Reference Cell and then apply them to commercial systems. ${ }^{7,8}$ Researchers who begin to understand the complex nature of the plasma discharge in a GEC reactor cannot necessarily infer knowledge about a commercial system unless the GEC cell is shown to behave like a commercial system. The goal of this research was to determine under what conditions the GEC cell operates like a typical commercial system. From there understanding about commercial systems can be inferred by experiments performed on the GEC cell in the parameter range where the GEC cell and commercial systems operate in a similar fashion.

In this paper, the performance of the original design of the GEC as a capacitively coupled discharge is compared to the performance of a commercial tool manufactured by SEMI Group, model 1000 TP/CC, a capacitively coupled discharge. Silicon wafers were etched using $\mathrm{CF}_{4}$ as the feed gas. We chose a $\mathrm{CF}_{4} / \mathrm{O}_{2}$ mixture because it is probably one of the most studied etching chemistries ${ }^{3}$ and because it is commonly used in our Solid State Electronics Laboratory (SSEL). In the SSEL, the SEMI Group etcher's primary purpose was to produce new devices, and so we felt that it was important to try to use it under normal operating conditions. The energetic electrons in the rf discharge dissociate the $\mathrm{CF}_{4}$ into $\mathrm{CF}_{x}$ (where $x$ can be 2 or 3 typically) and free $\mathrm{F}$ atoms. One of the most important aspects of fluorine chemistries is that $\mathrm{F}$ and the surface of the wafer react to produce a volatile
TABLE I. A comparison of the SEMI Group RIE and the GEC reference cell.

\begin{tabular}{lll}
\hline \hline & SEMI Group & GEC Cell \\
\hline Powered Electrode & lower & lower \\
Insulator Material & ceramic & Teflon \\
Upper Electrode & 16 in. showerhead & 4 in. showerhead \\
Lower Electrode & 12 in. & 4 in. \\
Electrode Spacing & 1 in. -6 in. & $1 \mathrm{in.}$ \\
Chamber diameter & $17 \mathrm{in.}$ & $10 \mathrm{in}$. \\
Frequency & $13.56 \mathrm{MHz}$ & $13.56 \mathrm{MHz}$ \\
Flowrate & $30 \mathrm{sccm}$ & $30 \mathrm{sccm}$ \\
Power & $44-176 \mathrm{~W}$ & $5-20 \mathrm{~W}$ \\
Pressure & $\sim 75-150 \mathrm{mT}$ & $\approx 75-150 \mathrm{mT}$ \\
Plasma Volume & fills entire chamber & mainly between electrodes \\
\hline \hline
\end{tabular}

reaction product, which can be easily pumped away. ${ }^{1-3}$ Note that at the pressures used in plasma etching, 1 mTorr to about $200 \mathrm{mTorr}$, the plasma is only $10^{-5} \%$ to $10^{-3} \%$ ionized. As mentioned, these few electrons, however, drive the chemical reactions by colliding with $\mathrm{CF}_{4}$.

\section{EXPERIMENT}

The two etching systems used in this study were the GEC reference cell and a SEMI Group 1000 TP/CC reactive ion etcher (RIE) housed in University of Michigan's Solid State Electronics Laboratory clean room (see Fig. 1). Both are parallel plate, rf systems with the bottom electrode powered and the upper electrode grounded. Table I gives a brief comparison of the two etchers. A major difference between the two systems is the size and spacing of the electrodes. The GEC reference cell has two 4 in. diameter aluminum electrodes, spaced 1 in. apart. Figure 2 shows a simplified schematic of the GEC reference cell. The design of the pumping port and related plumbing at the base of the GEC cell in particular has been simplified in this drawing. The plumbing is actually quite elaborate and the reduced pumping speed sets a lower limit on the operating pressure; see Ref. 9 for further details on the design of the GEC reference cell. The SEMI Group RIE was designed with an upper, grounded electrode of $16 \mathrm{in}$. and powered lower electrode of $12 \mathrm{in} . \mathrm{cm}$. The asymmetrical design of the electrodes allows the SEMI Group systems to develop the larger dc bias needed to produce fast etch rates. ${ }^{3}$ The two electrodes in the GEC cell have the same diameter. However, we set out to determine under what conditions the two systems would behave similarly despite this difference. The two systems are very similar in their design in that they are both parallel plate, capacitively coupled, rf diodes. It would be impossible to design one reference cell that represented the many commercial etcher designs. The GEC cell was carefully designed to emulate as many parameters of a commercial cell as possible. ${ }^{4}$

Gasses were introduced through the upper electrode for both systems in a showerhead design. The fluorine flow rate was regulated with mass flow controllers. On the GEC reference cell, needle valves were used to control the flow of gases. The pressure in the SEMI Group RIE and the GEC reference cell was monitored with a capacitive manometers. 


\section{GEC Reference Cell}

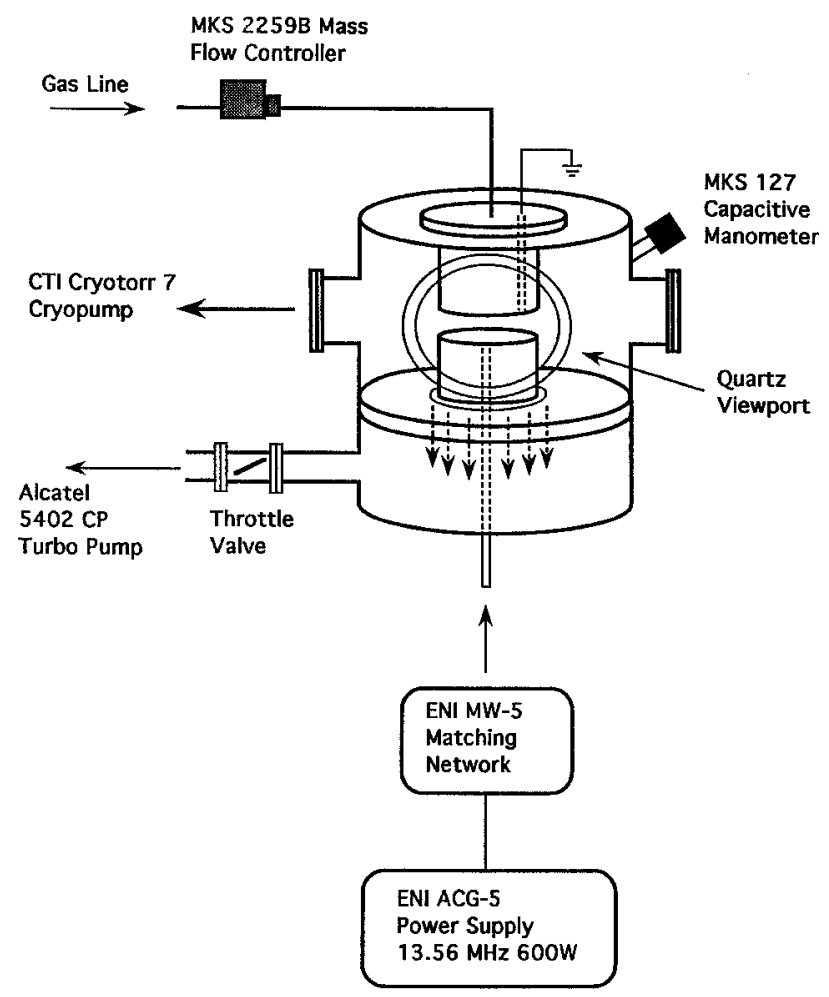

FIG. 2. Simplified schematic of the GEC reference cell.

In initial experiments to test whether the two systems could behave similarly, they both were used to etch identical silicon wafers in $\mathrm{CF}_{4} / \mathrm{O}_{2}$. The ex situ measured etch rates for both systems were compared. The etch rate is calculated by measuring the thickness of the wafer before and after the etch, using a scanning profilometer, (accuracy of $100 \AA$ as given by the manufacturer), and dividing by the etch time.

To obtain similar discharges in both systems, it was necessary for the electrical conditions between the plates to be matched as closely as possible. One solution that was considered was to match the ratio of the electrode area to electrode spacing to simulate a simple capacitor. The GEC cell has 4 in. diameter electrodes with a fixed, 1 in. plate spacing. To obtain the same ratio in the SEMI Group RIE would have required a plate space of over 8 in. The design of the SEMI Group made this a physical impossibility. Instead, the plate spacing was adjusted in the SEMI Group system to be 1 in., the same electrode spacing as the GEC cell. Figure 3(a) shows the etch rate in each plasma system as a function of power in a $\mathrm{CF}_{4} / \mathrm{O}_{2}$ plasma at $150 \mathrm{mT}$. It is apparent that the two systems show different slopes in the etch rate as the power is increased. The etch rate for the GEC cell increased more rapidly with power than the commercial system because the GEC cell has a much smaller volume of plasma. So the power density of the GEC cell was much larger for the same amount of input power to the two systems. The power density is what controls the amount of ionization, electron heating, and dissociation of the feed gas.
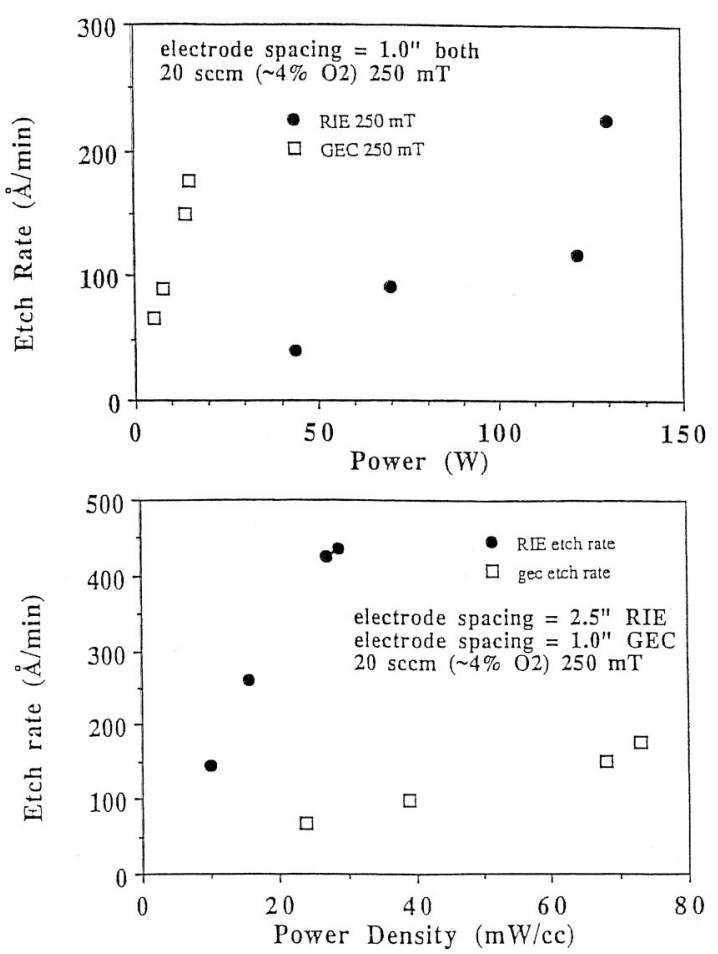

FIG. 3. (a) Etch rate of polysilicon vs power with 1 in. electrode spacing in both cells. (b) Etch rate vs power density with unequal spacing.

So, in order to have similar discharges in the two systems, it is necessary to achieve similar power densities in the plasma. ${ }^{10}$ To investigate the effect of input power on etching conditions, the etch rate in the two systems was examined as a function of power density under normal operating conditions. The GEC had a fixed electrode spacing of $1 \mathrm{in}$. and the SEMI Group is usually used with an electrode spacing of 2.5 in. (If the electrode spacing is too close, sometimes odd filaments between the two electrodes form and the discharge is no longer uniform.) The results are shown in Fig. 3(b). It is clearly seen that the two systems have very different discharges driving the etching. The commercial system had a much larger volume of plasma above the wafer compared to the GEC, even though the power densities were the same. Since the same size wafer was used in both systems, probably more fluorine atoms were available for reaction in the larger volume of discharge in the commercial system compared to the GEC cell. The net result was a faster etch rate in the commercial system.

Attempts were made to obtain a better match between the two systems by adjusting the gas flow in the two systems. Since the geometry of the two cells is quite different, the gas residence times are therefore also different. Etches performed as the gas flow was varied showed the etch rate to be largely unaffected by the change in residence time. This is probably due to the fact that the etches were not occurring in a regime where reactions at the surface of the wafer were "starved" for fluorine, i.e., excess fluorine was present.

When the power densities, i.e., ability to ionize the gas, heat the free electrons, and dissociate the feed gas were the same and the plate spacing, i.e., the amount of plasma above 

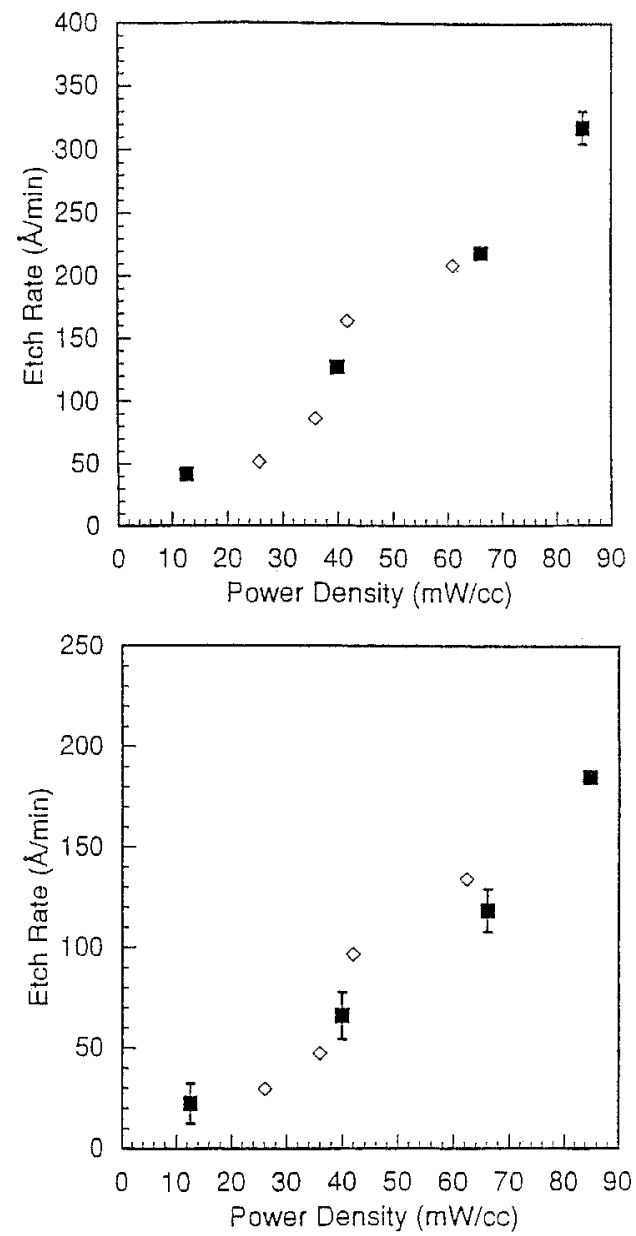

FIG. 4. (a) Etch rate as a function of absorbed power at $75 \mathrm{mT}$ for the GEC cell ( $\square$ ) and the SEMI Group RIE (ם) at 1 in. electrode spacing. (b) Etch rate as a function of absorbed power at $150 \mathrm{mT}$ for the GEC cell $(\diamond)$ and the SEMI Group RIE (ם) at 1 in. electrode spacing.

the wafer were the same, the two reactors behaved similarly; see Figs. 4(a) and 4(b).

This initial comparison of the first GEC reference cell involved measuring the current and voltage as close as possible to the bottom electrode of the GEC cell ${ }^{4}$ and assuming an input power to the SEMI Group as measured from the power supply. Accurate measurements of the power delivered to and absorbed by the discharge is important to many researchers and commercial manufacturers of plasma tools and many methods have been attempted with varying degrees of success. ${ }^{11-14}$

The power absorbed by the discharge produced by the SEMI Group is usually assumed by devices makers to be the

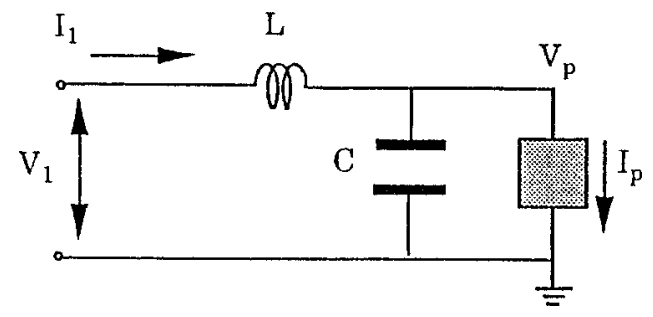

FIG. 5. Equivalent circuit model for the SEMI Group RIE.
75 mTorr

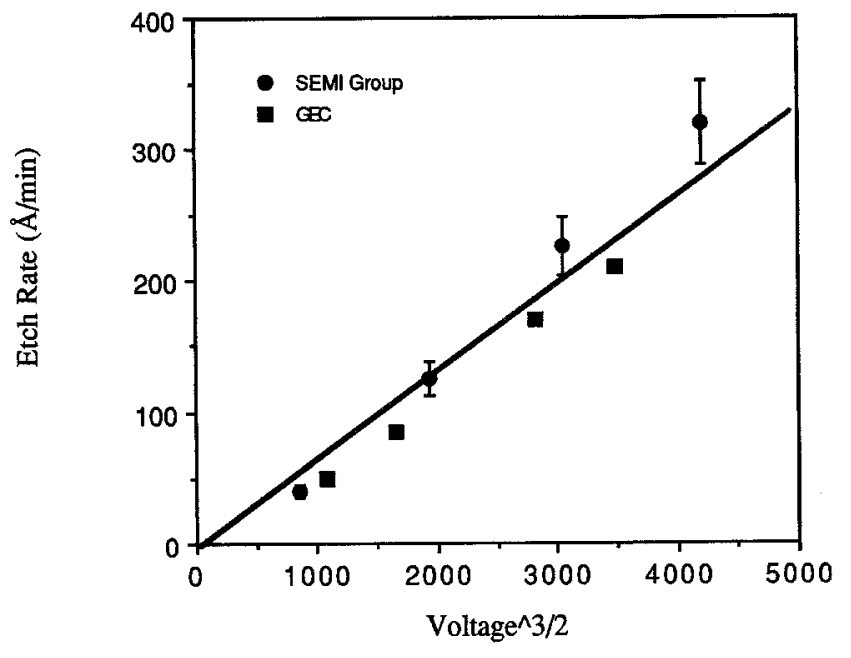

150 mTorr

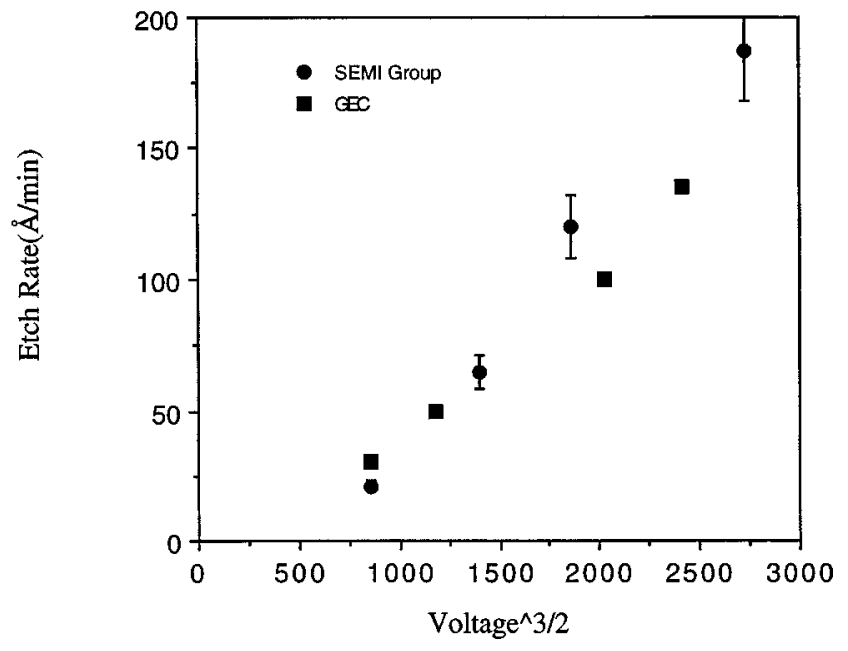

FIG. 6. (a) Etch rate as a function of bias voltage at $75 \mathrm{mT}$ for the GEC cell (西) and the SEMI Group RIE (-) at 1 in. electrode spacing. (b) Etch rate as a function of bias voltage ${ }^{3 / 2}$ at $150 \mathrm{mT}$ for the GEC cell (ם) and the SEMI Group RIE (-) at 1 in. electrode spacing.

value read from the meter on the power supply. However, the current can take many paths, so that the power delivered to the plasma is probably much smaller than the value read from the power supply. For this reason, it was necessary to determine the power absorbed by the plasma. This can be accomplished by modeling the discharge system and matching network as an equivalent circuit and solving the relevant equations once the capacitance and inductance of the system are measured. ${ }^{4}$ [Note that in $\mathrm{rf}$ discharge systems, the rf power supply is connected to a matching network which will automatically adjust the inductance and capacitance of the circuit for the change in the load when the gas (an open circuit) suddenly becomes a plasma (a short).] On the SEMI Group cell, there was no way to nonintrusively probe the current and voltage near the electrode, as was possible on the GEC cell. Therefore initially it was difficult to compare current and voltage measurements between the two systems. The GEC cell was designed so that the current and voltage 

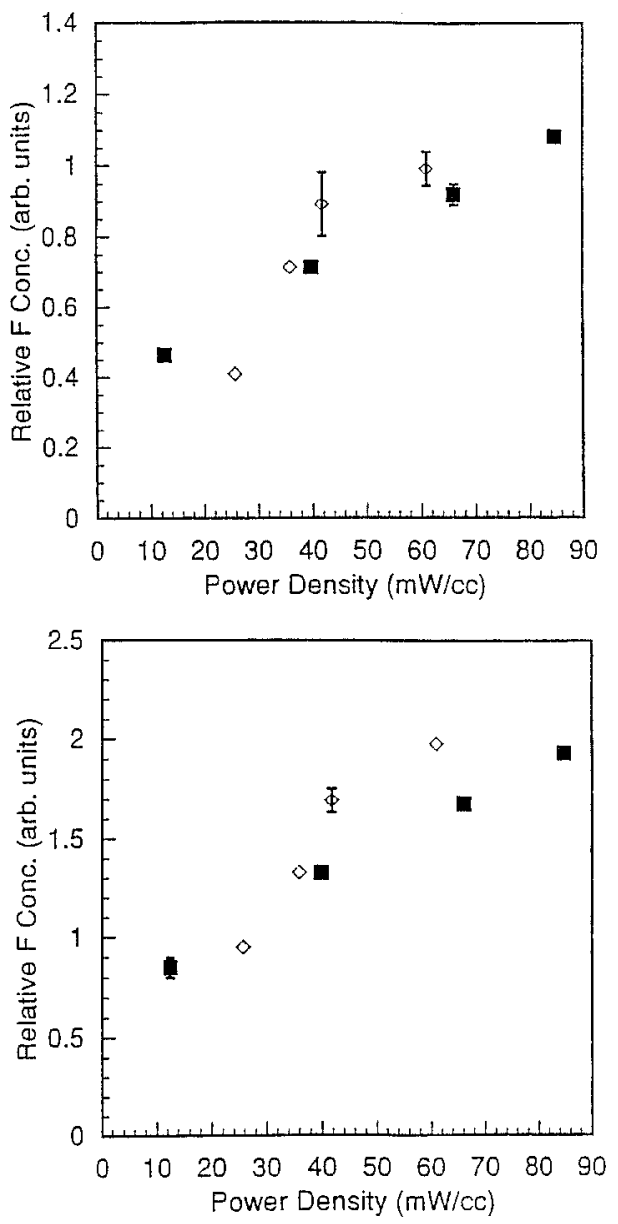

FIG. 7. (a) Relative fluorine concentration as a function of absorbed power at $75 \mathrm{mT}$ for the GEC cell $(\diamond)$ and the SEMI Group RIE (ם) at 1 in. electrode spacing. (b) Relative fluorine concentration as a function of absorbed power at $150 \mathrm{mT}$ for the GEC cell $(\diamond)$ and the SEMI Group RIE (ם) at 1 in. electrode spacing.

could be measured very near the spot where the rf voltage was applied to the electrode. In the SEMI Group system, however, the only assessable spot turned out to be at the cell side of the matching network. The probes at this point sees a 30.5 in. RG-220/U Time Microwave Communications coaxial cable in series with the etching chamber.

To determine the power absorbed by the plasma in the SEMI Group discharge the system was modeled as a coaxial cable in series with a capacitor (the net capacitance of the SEMI Group RIE). See Ref. 15 for details of the model. The power absorbed by the plasma in the GEC cell was calculated with a similar, but slightly different circuit model. ${ }^{16}$ This model was coded into a small computer program by Paul Miller (Sandia). ${ }^{16}$ The input parameters, besides the capacitance and inductance of the system, are the applied voltage and the measured current at the bottom electrode. All of the groups involved in the initial testing of the GEC cell used Miller's code. 4

The net capacitance of the SEMI Group RIE in series with the coaxial cable capacitance was calculated to yield a total capacitance of $423 \mathrm{pF}$. This agrees well with a BK Precision capacitance meter measurement of approximately $450 \mathrm{pF} \pm 50 \mathrm{pF}$. The total capacitance taken with the coax inductance gives a resonant frequency of $17.4 \mathrm{MHz}$, which is very close to the measured resonance.

The model verified that the SEMI Group RIE could be represented by the equivalent circuit shown in Fig. 5 . This is the same circuit model as was used for the GEC cell ${ }^{16}$ only now $L$ is the inductance of the coaxial cable and $C$ is the combined capacitance of the coaxial cable and the etching chamber. The equations used to determine the power delivered to the plasma of the GEC could be used to determine the absorbed by the discharge in the SEMI Group RIE. Minor changes were made to some of the equations because the resonance of the cell now occurred between the first and second harmonic instead of between the second and third as was seen with the GEC reference cell. ${ }^{4}$

\section{ETCHING RESULTS}

A group of experiments were conducted which compared the etch rate, the fluorine concentration from actinometry, and the measured bias voltage as a function of calculated power density. The current in both systems was measured with a Pearson current probe and a high voltage probe was used to measure the voltage. Using the model described in Refs. 15 and 16, the power density of the plasma was calculated from the current and voltage measurements. Figures 4(a) and 4(b) show the etch rate as a function of power density. There is a reasonable agreement between the results of the SEMI Group RIE and the GEC cell. Although both systems exhibited similar etch rates, the SEMI Group consistently etched a little faster, probably due to the electrode asymmetry. As previously mentioned, the conditions on the SEMI Group RIE were altered to match the plate spacing and power densities of the GEC reference cell. This did not correct for the higher bias voltages that tended to form in the SEMI Group RIE. The bias voltage (as previously discussed) was recorded, although there is no method to independently control the bias voltage. It depends upon the system design and the power delivered from the power supply.

At higher pressures and lower powers, the etching is in a more chemically driven regime where the ion impact energy at the surface, and therefore the bias voltage, is not as important, ${ }^{17}$ see Eq. (1). In this regime the two systems matched very well. In general, the etch rate is a combination of physical sputtering and chemical reactions of $\mathrm{F}$ atoms with the surface. At low pressures, the concentration of fluorine will be small and sputter etching will dominate. This effect can be seen by comparing a 75 mTorr case (the lowest we were able to run) and a 150 mTorr case. As shown by Fig. 6(a), where the etch rate is plotted as a function of $V^{3 / 2}$, the data can be approximated by a straight line as predicted by Eq. (1). But in Fig. 6(b), at a pressure of 150 mTorr, the etching is driven by chemical reactions, as well as physical sputter etching, compared to the 75 mTorr case and the data does not fit a straight line through the origin. Equation (1) describes the ion flux, which predicts the amount of ions that are accelerated across the sheath. At 75 mTorr, chemical reactions are undoubtedly occurring, but to a lesser extent than at 150 mTorr. 
The fluorine concentration was monitored with a technique called actinometry. ${ }^{18,19}$ With this technique, the relative concentration of the ground state of a reactive specie can be determined by monitoring the ratio of the optical line emission from that species and the optical emission of an inert "actinometer," in this case argon, if certain necessary conditions are met. ${ }^{18,19}$ Therefore, $[\mathrm{F}] \sim[\mathrm{Ar}] I_{\mathrm{F}} / I_{\mathrm{Ar}}$ where the concentration of argon $[\mathrm{Ar}]$ is kept constant and $I_{\mathrm{F}}$ is the intensity of a neutral fluorine line, in this case $703.75 \mathrm{~nm}$ and $I_{\mathrm{Ar}}$ is the intensity of an neutral argon line, in this case $705.02 \mathrm{~nm}$. Results similar to the etch rate studies versus power density were observed when the etch rate as a function of the relative fluorine concentration [Figs. 7(a) and 7(b)] was compared. The fluorine concentration in the GEC cell and the SEMI Group RIE are very similar as a function of power.

\section{CONCLUSION}

Etching conditions in the GEC reference cell were compared to a SEMI Group 1000 TP/CC reactive ion etcher. To achieve similar etching conditions in both systems, the plate spacing, pressure, and power density need to be as similar as possible. A comparison of the etching results and the relative fluorine concentration as a function of bias voltage and power density show that the GEC cell can emulate the performance of a commercial system. With these results in mind, new sensor and diagnostics developed on the GEC cell can be transferred to commercial cells and under the right conditions, similar performance can be expected.

\section{ACKNOWLEDGMENTS}

We would like to acknowledge funding from the Semiconducting Research Corporation. We would also like to thank Ward Getty for his technical advice and insight.

${ }^{1}$ D. M. Manos and D. L. Flamm, Eds. Plasma Etching (Academic, San Diego, 1989).

${ }^{2}$ M. Lieberman and A. Lichtenberg, Principles of Plasma Discharges and Materials Processing (Wiley, New York, 1994).

${ }^{3}$ Brian Chapman, Glow Discharge Processes (Wiley, New York, 1980).

${ }^{4}$ P. J. Hargis, K. E. Greenberg, P. A. Miller et al., Rev. Sci. Instrum. 65 , 140 (1994).

${ }^{5}$ M. L. Brake, J. Pender, M. Buie et al., J. Res. Natl. Inst. Stand. Technol. 100, 441 (1995).

${ }^{6}$ P. A. Miller, G. A. Hebner, K. E Greenberg et al., J. Res. Natl. Inst. Stand. Technol. 100, 427 (1995).

${ }^{7}$ S. Shannon, J. P. Holloway, K. Flippo, and M. L. Brake, Appl. Phys. Lett. 71, 1467 (1997).

${ }^{8}$ M. Buie, J. Pender, J. Holloway, and M. Brake, IEEE Trans. Plasma Sci. 24, 111 (1996).

${ }^{9}$ M. Passow, Ph. D. Thesis, University of Michigan, Ann Arbor, MI (1991).

${ }^{10}$ A. T. Bell, J and EC Fundamentals 9, 160 (1970).

${ }^{11}$ J. W. Butterbaugh, L. D. Baston, and H. H. Sawin, J. Vac. Sci. Technol. A 8, 916 (1990).

${ }^{12}$ B. Andries, G. Ravel, and L. Peccoud, J. Vac. Sci. Technol. A 7, 2774 (1989).

${ }^{13}$ V. A. Godyak and R. B. Piejak, J. Vac. Sci. Technol. A 8, 3833 (1990).

${ }^{14}$ W. G. M. Van den Hoek, C. A. M. Vries, and M. G. J. Heijman, J. Vac. Sci. Technol. B 5, 647 (1987).

${ }^{15}$ J. T. P. Pender, Ph.D. Thesis, University of Michigan, Ann Arbor, MI (1995).

${ }^{16}$ P. A. Miller, private communication (1990).

${ }^{17}$ J. Ding et al., J. Vac. Sci. Technol. A 11, 1283 (1993).

${ }^{18}$ J. W. Coburn and M. J. Chen, J. Appl. Phys. 51, 3134 (1980).

${ }^{19}$ R. A. Gottscho and V. Donnelly, J. Appl. Phys. 56, 245 (1984). 